\title{
SALVIA TILANTONGENSIS (LAMIACEAE), UNA ESPECIE NUEVA DE LA MIXTECA ALTA DE OAXACA, MÉXICO
}

Jesús Guadalupe GonZÁlez-Gallegos ${ }^{1,3}$ y Remedios Aguilar-Santelises ${ }^{2}$

\author{
${ }^{1}$ Universidad de Guadalajara, Departamento de Botánica y Zoología, Herbario \\ "Luz María Villarreal de Puga" del Instituto de Botánica (IBUG), km 15.5 carretera \\ Guadalajara-Nogales, Las Agujas, 45110 Zapopan, Jalisco, México. \\ ${ }^{2}$ Instituto Politécnico Nacional, Centro Interdisciplinario de Investigación \\ para el Desarrollo Integral Regional, calle Hornos 1003, \\ 71230 Xoxocotlán, Oaxaca, México. \\ 33Autor para la correspondencia: xanergo@hotmail.com
}

\section{RESUMEN}

Se describe e ilustra a Salvia tilantongensis como una especie nueva de la Mixteca Alta de Oaxaca, México. El taxon es similar en su morfología a Salvia fulgens y S. gesneriiflora. Se distingue de ambas por su corteza exfoliante, peciolos articulados en un engrosamiento del nudo, hojas más estrechas, brácteas florales de menor tamaño y de ápice más corto, pedicelos más largos durante la fructificación, los lóbulos del cáliz agudos y largamente aristados, lóbulo medio del labio inferior de la corola incurvado cóncavo, filamento y estilo más cortos, y rama inferior del estilo más larga. Además, difiere de S. fulgens por la ausencia de un par de papilas en la porción basal del tubo de la corola. También se provee de descripciones detalladas de $S$. fulgens y $S$. gesneriiflora.

Palabras clave: endemismo, Salvia fulgens, Salvia gesneriiflora, sección Fulgentes, sección Nobiles.

\section{ABSTRACT}

Salvia tilantongensis is described and illustrated as a new species from the Mixteca Alta of Oaxaca, Mexico. The new taxon is morphologically similar to Salvia fulgens and $S$. gesneriiflora. It differs from both by its exfoliating bark, petioles attached to a thickened base from the node, narrower leaves, smaller floral bracts and with a shorter apex, longer 
pedicels during fructification, acute and long aristate lobes of the calyx, middle lobe of lower corolla lip incurved-concave, shorter filament and style, and longer lower stigmatic branch. Furthermore, it is distinguished from $S$. fulgens by the absence of a pair of papillae inside the basal portion of the tube of the corolla. Detailed descriptions of S. fulgens and $S$. gesneriiflora are also provided.

Key words: endemism, Salvia fulgens, Salvia gesneriiflora, section Fulgentes, section Nobiles.

El género Salvia L. es uno de los más diversos dentro de la familia Lamiaceae, con 900 a 1000 especies a escala global (Standley y Williams, 1973; Harley et al., 2004). Exhibe una distribución casi cosmopolita, no tiene representantes silvestres únicamente en Australia y las regiones más frías de ambos hemisferios. Sus principales centros de diversificación se encuentran en México, la cuenca del Mediterráneo, China y Medio Oriente (Harley et al., 2004; Walker et al., 2004). La morfología particular del androceo de sus flores (un par de estambres con conectivo alargado que separa a las dos tecas, la posterior frecuentemente reducida o ausente y el conectivo modificado en una especie de timón) la distingue, y es clave durante la polinización de las flores del género (Claßen-Bockhoff et al., 2003; Walker et al., 2004; Walker y Sytsma, 2007; Wester y Claßen-Bockhoff, 2006a, 2006b, 2007a, 2007b, 2011). Sin embargo, la monofilia de Salvia y su delimitación tradicional (Bentham, 1832, 1848, 1876; Briquet, 1897) han sido cuestionadas ante los resultados obtenidos de análisis filogenéticos con base en secuencias de ADN, los cuales han demostrado que se trata de un grupo polifilético debido a que otros géneros (Dorystaechas Boiss. \& Heldr. ex Benth., Meriandra Benth., Perovskia Kar., Rosmarinus L. y Zhumeria Rech. f. \& Wendelbo) han derivado de antecesores dentro del linaje de Salvia (Walker et al., 2004; Walker, 2006; Walker y Sytsma, 2007; Jenks et al., 2011; Jenks et al., 2013). Esto demanda la necesidad de una recircunscripción de los componentes de este género en el futuro próximo, pero de momento se sigue respetando la delimitación tradicional.

México, con alrededor de 300 especies, es el país que alberga la mayor riqueza de Salvia (Ramamoorthy y Elliott, 1998; Villaseñor, 2004; Martínez-Gordillo et al., 2013). Casi la totalidad de las especies de nuestro país, incluidos los taxones que se describen en este documento, pertenecen al subgénero Calosphace (Benth.) Epling. Sin embargo, también existen representantes de los subgéneros Heterosphace (Salvia henryi A. Gray, S. roemeriana Sheele y $S$. summa A. Nelson), y Salviastrum ( $S$. 
texana (Sheele) Torr. y $S$. whitehousei Alziar), y de la sección Audibertia (S. apiana Jeps., S. brandegeei Munz, S. californica Jeps., S. carduacea Benth., S. clevelandii (A. Gray) Greene, S. columbariae Benth., S. mohavensis Greene, S. munzii Epling, S. pachyphylla Epling ex Munz y S. vaseyi (Porter) Parish) (Epling, 1938; Strachan, 1982; Neisess, 1983; Walker y Elisens, 2001; Martínez-Gordillo et al., 2013). Ramamoorthy y Elliott (1998) reconocieron, con base en referencias bibliográficas y datos inéditos del primer autor, que el estado de Oaxaca contiene la mayor cantidad de especies al poseer una cifra de 63. En esa misma publicación señalaron 35 especies para Chiapas y 48 para Michoacán. Sin embargo, revisiones puntuales recientes para estas dos últimas entidades revelan una cantidad mayor para cada una de ellas, 84 para Oaxaca (García-Mendoza y Meave, 2011), 75 para Chiapas (DomínguezVázquez et al., 2002) y 73 (Rodríguez-Jiménez y Espinosa-Garduño, 1996) o 64 (Cornejo-Tenorio e Ibarra-Manríquez, 2011) para Michoacán. El incremento en el número de especies es contundente, lo que señala que persiste un conocimiento insuficiente del género en el país. Es de esperar que el desarrollo de investigaciones y exploración botánica resulten en el descubrimiento de taxones en lugares donde no hayan sido registrados antes, y de algunos aún no descritos. En este sentido, aquí se presenta y describe una de estas nuevas adiciones: Salvia tilantongensis, especie que es semejante en su morfología a Salvia fulgens Cav. y S. gesneriiflora Lindl. \& Paxton; éstas son a la vez parecidas entre sí y confundidas con frecuencia, por lo que en este documento se ofrece primero una descripción detallada de ellas, para luego contrastarlas con $S$. tilantongensis.

La especie fue evidenciada durante el desarrollo de un estudio florístico en el municipio de Santiago Tilantongo, Oaxaca; una vez que se determinó que se pudiera tratar de un taxon no descrito, se procedió a revisar especímenes de herbario de las especies más afines. Las colecciones consultadas fueron: CHAPA, CIIDIR, CIMI, CREG, ENCB, GUADA, HUAA, HUMO, IBUG, IEB, MEXU, MICH, OAX, UC, WIS y ZEA. La información de las etiquetas de los especímenes examinados fue capturada en una base de datos. Se estimaron las coordenadas geográficas para aquellos registros que carecían de ellas de acuerdo con los criterios de Wieczorek (2001) pero sin el cálculo de radios de incertidumbre. Con base en lo anterior, se elaboraron mapas con los trazos individuales de cada taxon para ilustrar su distribución. A continuación se presentan los resultados:

Salvia fulgens Cav., Icon. 1: 15, t. 23. 1791. Tipo: Icon. 1: 1, t. 23 (lectotipo: ilustración depositada en el herbario MA, designado por Ramamoorthy, 1984). Fig. 1A. Salvia cardinalis Kunth, Nov. Gen. Sp. (quarto ed.) 2: 301, t. 152. 1818. 
Salvia boucheana Kunth, Index Sem. (Berlin): 9. 1845.

Salvia incana M. Martens \& Galeotti, Bull. Acad. Roy. Sci. Bruxelles 11: 68. 1844. Salvia orizabensis Fernald, Proc. Amer. Acad. Arts 35(25): 538. 1900.

Hierba perenne, sufruticosa, de 0.8 a 2(-4) $\mathrm{m}$ de alto, erecta y en ocasiones subescandente; tallos pilosos y puberulentos, con frecuencia cubiertos con tricomas glandular-capitados cortos. Hojas con peciolo de 1.1 a $5.0(-7.0) \mathrm{cm}$ de largo, piloso y en ocasiones con tricomas glandular-capitados cortos; lámina ovado-lanceolada a ovada, de (1.5-)4.6 a 10.0(-14.0) × 2.0 a 3.3(-7.0) cm, aguda en el ápice, cordada, oblicua, truncada a redondeada en la base, serrada a serrulada en el margen, pilosa o con tricomas adpresos en el haz, pilosa o con menor frecuencia tomentosa en el envés. Inflorescencia terminal, de 9.5 a $30 \mathrm{~cm}$ de largo, con 4 a 8 verticilastros, cada uno con 2 a 8 flores, los de la base separados entre sí por 1.5 a $2.6 \mathrm{~cm}$, eje floral piloso y con tricomas glandular-capitados cortos. Bráctea floral ovado-lanceolada, de (1.9-) 2.0 a $3.6(-4.3) \times 0.6$ a $1.0 \mathrm{~cm}$, decidua, verde o roja, truncada en la base, largamente atenuada en el ápice (9.0 a $19.6 \mathrm{~mm}$ ), margen entero, pilosa en la cara externa y en ocasiones con tricomas glandular-capitados cortos. Flores con pedicelos de 4.0 a 8.0 $\mathrm{mm}$ de largo (hasta $11.3 \mathrm{~mm}$ en fruto), pilosos y con tricomas glandular-capitados cortos. Cáliz de 1.4 a $1.8 \mathrm{~cm}$ de largo, de 0.6 a $0.9 \mathrm{~cm}$ de ancho en la garganta (alcanzan hasta $2 \times 1.2 \mathrm{~cm}$ en fruto), verde o rojo, piloso o hispídulo en el exterior y con tricomas glandular-capitados cortos, hispídulo a glabro en el interior y hacia los labios, lóbulos agudos, el superior entero, con 5 o 7 venas. Corola de color rojo brillante, pilosa en el labio superior y en el dorso de la porción media distal, o en algunas ocasiones con algunos tricomas dispersos a todo lo largo; tubo de 2.5 a 3.6(-3.9) cm de largo, ventricoso, de 0.7 a $0.9(-1.0) \mathrm{cm}$ de ancho en el vientre, recto en la base y ornamentado con un par de papilas en el interior; labio superior de 1.6 a 1.9(-2.1) cm de largo, labio inferior de 1.4 a $1.8 \times(0.4-) 0.6$ a $1.0(-1.2) \mathrm{cm}$, lóbulo medio reflejo. Estambres insertos en la corola; filamento de 4.8 a 7.4(-8.3) $\mathrm{mm}$ de largo; conectivo de 2.2 a $2.8 \mathrm{~cm}$ de largo, recto, con un diente corto y retrorso después de la inserción del filamento hacia la porción posterior, o bien sin ornamentaciones, piloso en el dorso arriba de la inserción del filamento; teca de 3.0 a 3.8(-4.2) mm de largo; un par de estaminodios filiformes ubicados atrás y arriba de la inserción del filamento a la corola. Cuerno ginobásico de 0.9 a $1.6 \mathrm{~mm}$ de largo; estilo de 4.0 a $5.3(-5.6) \mathrm{cm}$ de largo, piloso hacia el ápice, en las caras dorsal y ventral, rama superior arqueada, (2.5-)3.9 a $8.9 \mathrm{~mm}$ de largo, rama inferior aguda y recta, (1.3-)2.1 a $3.7 \mathrm{~mm}$ de largo. Mericarpio ovoide, de 3.0 a $4.1 \times 1.9$ a $2.5 \mathrm{~mm}$, café oscuro uniforme o café claro y jaspeado con un tono más oscuro, liso y glabro. 
González-Gallegos y Aguilar-Santelises: Salvia tilantongensis, especie nueva de Oaxaca, México
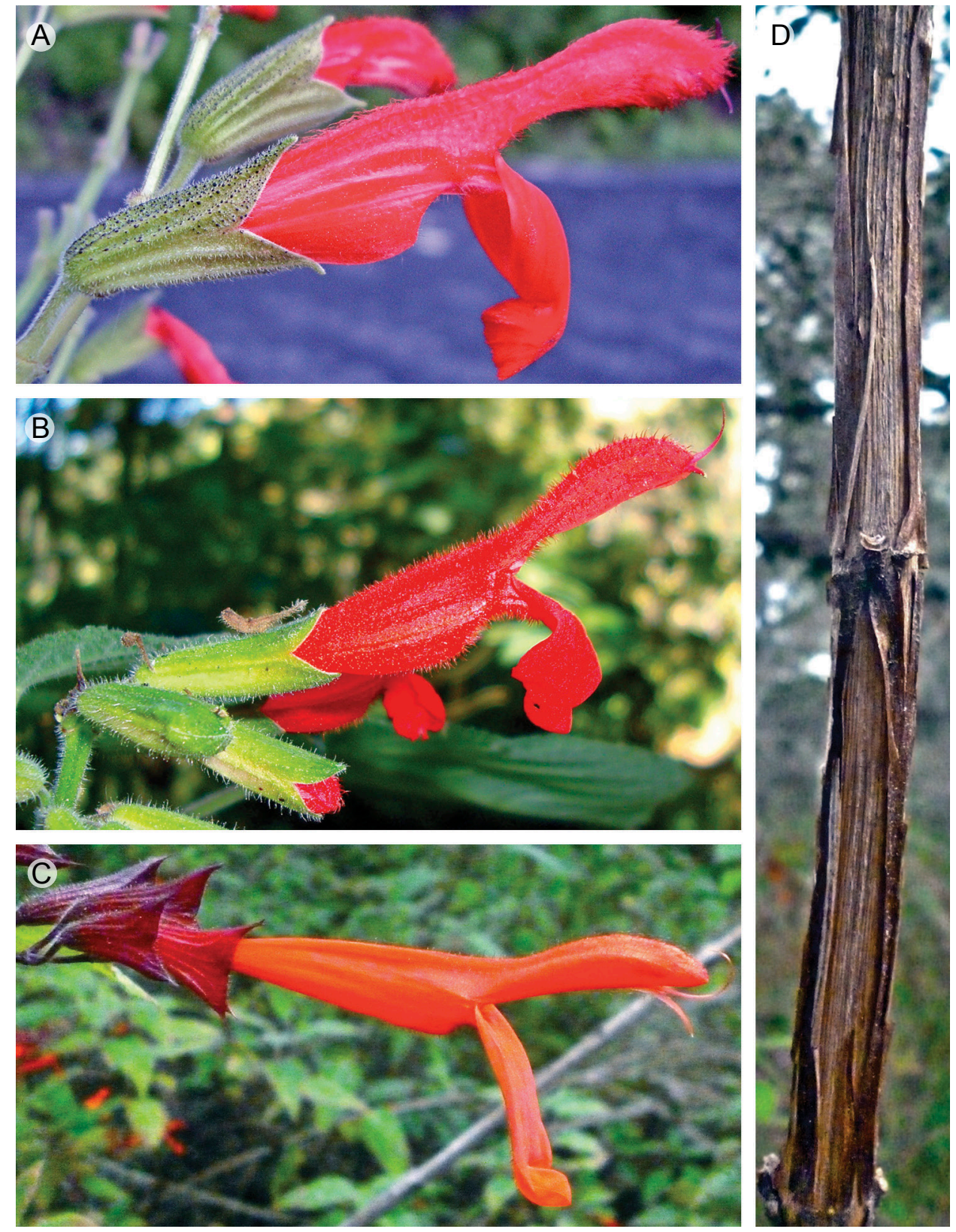

Fig. 1. Flores de A. Salvia fulgens, B. S. gesneriiflora y C. S. tilantongensis sp. nov. El inciso D. muestra el tallo exfoliante de esta última (fotografías tomadas por J. G. GonzálezGallegos. A. en Acuitzio del Canje, Michoacán; B. en Sierra de Manantlán, Jalisco; C y D. en Santiago Tilantongo, Oaxaca). 
Material selecto examinado: MÉXICO, Distrito Federal. Álvaro Obregón: San Bartolo, 2700 m, 17 jul 1977, A. Ventura-A. 2937 (MEXU); Magdalena Contreras: Llanos de Copilco, al SE del Tercer Dínamo, 10 dic 1983, J. L. Villaseñor-R. \& E. Martínez-S. 633 (MEXU); Cuajimalpa: Área Natural Protegida Desierto de

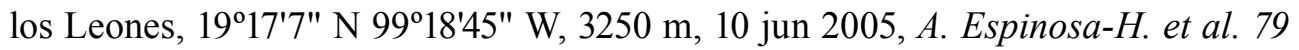
(MEXU); Milpa Alta: Volcán Tláloc, ladera E, 3200 m, 23 jul 1986, G. Zamudio-V. 15 (MEXU); Tlalpan: Topilejo, 2650 m, 27 nov 1976, A. Ventura-A. 2425 (MEXU). Estado de México. Amecameca: $12 \mathrm{~km}$ al E de Amecameca, 14 nov 1976, A. MarínM. 183 (IBUG, MEXU); Chalco: cerca de la colonia Agrícola Manuel Ávila Camacho, 2750 m, 11 sep 1966, A. Vargas-N. s.n. (MEXU); $2 \mathrm{~km}$ al N de San Pedro, cerca de Amecameca, 2680 m, 26 ago 2000, M. Crone 15 (OAX); Iturbide: alrededores de la Presa de Iturbide, 28 nov 1982, C. García-G. 130 (IBUG, IEB, MEXU, OAX); Ixtapaluca: campo experimental de investigación y enseñanza Zoquiapan, $3300 \mathrm{~m}$, 8 jul 1978, J. García-P. 12923 (GUADA); Naucalpan: Villa Alpina, 3200 m, 7 nov 1979, B. J. Guillermo-R. 69 (CIIDIR, MEXU); San Felipe: Palo Amarillo, 3100 m, 28 jul 1984, A. Hernández-F. et al. 91(MEXU); San José de Allende: $71 \mathrm{~km} \mathrm{~W}$ of Toluca near hwy 15, $26 \mathrm{~km}$ E of Zitácuaro, near the Michoacán border, 19²4' N $100^{\circ} 10^{\prime}$ W, 2700 m, 11 nov 1985, S. A. Reisfield 1260 (MEXU); Texcoco: Santa Catarina del Monte, 15 nov 1983, E. Ventura-V. 1604 (IBUG, IEB); Tlalmanalco: aproximadamente $3 \mathrm{~km}$ al E de San Rafael, $2700 \mathrm{~m}$, 19 sep 1976, P. G. García-G. 457 (IEB, MEXU); Zinacantepec: 2 km S de Raíces, Nevado de Toluca, 19¹1'27" N 99²4'28" W, 3262 m, 15 jul 2002, J. Martínez-R. et al. 22 (MEXU); Villa de Allende: en el ejido Cuesta del Carmen, cerca del límite entre Michoacán y México, 2830 m, 2 abr 1985, J. C. Soto-N. et al. 7920 (MEXU). Guerrero. Coyuca de Catalán: 80 km por la carretera de Ixtapa-Zihuatanejo rumbo a Coyuca de Catalán (Ciudad Altamirano), 1758'53.3" N 101²12'22.3" O, 1751 m, 26 sep 2010, J. G. González-Gallegos et al. 731 (IBUG). Hidalgo. Huasca de Ocampo: San Miguel Regla, 27 sep 1987, E. Argüelles 1981 (MEXU). Michoacán. Angangueo: Estación Chincua, Reserva de la Biósfera Mariposa Monarca, 19³9'75" N 100¹6'34" W, 3190 m, 4 ago 2000, M. G. Cornejo-T. 19 (IEB, MEXU); Contepec: cerro Altamirano, $2 \mathrm{~km}$ de Contepec, $20^{\circ} 0$ '21" N 1007'34" W, 2400 m, 15 sep 2005, M. G. Cornejo-T. \& M. A. SalinasM. 1579 (IBUG, IEB); Cuanajo: cerro del Burro, 20 oct 1985, J. M. Escobedo 513 (IBUG, IEB); Huiramba: parte alta del cerro de la Taza, 22 ago 1986, H. Díaz-B. \& $S$. Zamudio 2620 (CIIDIR, IBUG, IEB, MEXU); Los Reyes: aproximadamente 3 km al N de Pamatácuaro, 3080 m, 22 oct 1990, L. Torres-R. 491(ENCB, IEB); Morelia: parte alta del cerro del Águila, $3100 \mathrm{~m}, 19$ ago 1988, S. Zamudio 6679 (IEB, MEXU); Ocampo: El Rosario, 19³5'51" N 100¹5'59" W, 3230 m, 5 mar 2009, G. 
Cornejo-T. \& G. Ibarra-Manríquez 3383 (IEB, MEXU); Pátzcuaro: cerro El Frijol, 2900 m, 26 oct 1989, E. Pérez-C. 686 (IEB, MEXU); Quiroga: ladera sur del cerro Tzirate, 18 sep 1986, H. Díaz-B. \& S. Zamudio 2833 (IEB, IBUG, MEXU); Salvador Escalante: en el cerro Burro, $14 \mathrm{~km}$ al SE de Opopeo, $2820 \mathrm{~m}, 12$ oct 1985, J. C. Soto-N. 10782 (IEB, MEXU); Tancítaro: lado W del volcán de Tancítaro aprox. a 3 km al NE de El Jazmín, 29 sep 1989, I. García-R. \& J. Nava V. 2919 (CIMI, GUADA, IBUG, IEB); Tangancícuaro: aproximadamente $2 \mathrm{~km}$ al SW de Patamban, cerro Patamban, 2400 m, 1 abr 1989, L. Torres-R 221 (IEB); Tlalpujahua: ejido San Pedro Tarimbaro, 16 ene 1987, S. Zamudio \& N. López 5114 (IEB, IBUG); Zinapécuaro: Camino Viejo, hacia la presa La Gachupina, 2640 m, 11 oct 1984, M. J. Jasso 287 (IEB, MEXU); Zitácuaro: $20 \mathrm{~km}$ al NE de Zitácuaro, $2560 \mathrm{~m}, 28$ ene 1986, J. C. Soto-N. 11877 (MEXU). Morelos. Huitzilac: W of Lagunas de Zempoala guard station, near Morelos-Mexico state line, 2800 m, 10 ago 1981, D. Engle \& D. Remington 91 (MEXU). Puebla. Tehuacán de las Granadas, $5000 \mathrm{ft}, 1840$, H. Galeotti 643 (BR, P); Limestone hills near Tehuacán, ago 1897, C. G. Pringle 7449 (ENCB); Caltepec: vicinity of San Luis Tultilanapa, near Oaxaca, jun 1908, C. A. Purpus 3289 (UC); San Nicolás de los Ranchos: NE slope of Popocatépetl, above Xalitxintla, 3000 m, 16 oct 1968, H. Ern 372 (ENCB). Tlaxcala. Nanacamilpa: $2.5 \mathrm{~km}$ al W de San Felipe Hidalgo, 2700 m, 17 mar 1998, M. Agonizante 66 (MEXU). Veracruz: Orizaba, 1855 o 1856 (probablemente), M. Botteri $1168(\mathrm{GH})$.

Salvia fulgens se distribuye de manera predominante en la Franja Volcánica Transmexicana (Fig. 2). Crece en el Distrito Federal y en los estados de Guerrero, Hidalgo, México, Michoacán, Morelos, Puebla y Tlaxcala (Fig. 2). También se registra de las inmediaciones del Pico de Orizaba en Veracruz con base en una única colecta (M. Botteri 1168, GH); sin embargo, debido a que la localidad del ejemplar es imprecisa, no se muestra en el mapa de distribución (Fig. 2). Por otra parte, si se aceptara la inclusión de $S$. schaffneri como sinónimo de $S$. fulgens de acuerdo con Epling (1939), la distribución de esta última especie tendría que extenderse a San Luis Potosí. No obstante, aquí no se reconoce tal propuesta ya que existen algunas características que difieren entre ambos taxones, por ejemplo las brácteas florales en $S$. schaffneri alcanzan hasta $5 \mathrm{~mm}$ de largo, mientras en $S$. fulgens varían de (1.9-) 2.0 a 3.6(-4.3). Sin embargo, es necesario que $S$. schaffneri sea redescubierta y una mayor cantidad de especímenes disponibles para tomar una decisión clara sobre su estatus taxonómico.

Salvia fulgens crece en bosques de oyamel, de pino y encino, y de forma ocasional en bosque mesófilo de montaña; ocupa un intervalo de elevación de 2400 a 
$3400 \mathrm{~m}$, aunque en el Popocatépetl se ha registrado hasta a $3990 \mathrm{~m}$ (Barkley et al. 25, MEXU, UC). Por otra parte, los especímenes que corresponden a lo que se describió como S. incana (Galeotti 643 (BR, P), Pringle 7449 (ENCB) y Purpus 3289 (UC)) fueron colectados probablemente en matorral xerófilo en suelos calcáreos, un ambiente que contrasta con el hábitat en que se desarrolla el resto de poblaciones de $S$. fulgens. Epling (1939) propuso S. incana como sinónimo de S. fulgens, de la cual no difiera más que por presentar láminas foliares de 1.5 a $2.5 \mathrm{~cm}$ de largo, esta reducción de tamaño bien podría ser una adaptación al ambiente más árido en que crece; sin embargo, los especímenes de $S$. incana carecen de brácteas florales y la descripción de la estructura no está detallada en su protólogo, por tanto es deseable que se realice exploración botánica para documentar mejor la variación morfológica del taxon y caracterizar su hábitat. Esta especie suele formar poblaciones densas. La floración y fructificación son sincrónicas, pueden desarrollarse en cualquier momento del año, pero con mayor frecuencia de agosto a noviembre. De manera regular se le conoce como mirto, y los Mazahuas la nombran como tsímbarenzé; además se registra su uso como forraje (B. Farfán 40, IEB).

Este taxon es muy semejante en su morfología vegetativa y floral a Salvia gesneriiflora. Los gradientes de variación en hábito y porte de las plantas, forma, tamaño, indumento y color de las hojas, longitud de las inflorescencias, y tamaño, color y pubescencia de las flores, conforman un continuo de variación que dificulta su valor como caracteres diagnósticos. Debido a lo anterior, la distinción entre estos taxones ha sido problemática, es frecuente encontrar errores de determinación en ambos sentidos en especímenes de herbario y en los listados florísticos. No obstante estas especies pueden reconocerse con base en las siguientes características: a) longitud de las brácteas florales, en $S$. fulgens miden de (1.9-)2.0 a $3.6(-4.3) \mathrm{cm}$, mientras en $S$. gesneriiflora en general son de menor longitud con una variación que va de (0.6-)0.7 a 2.0(-2.4) $\mathrm{cm}, \mathrm{b}$ ) presencia de un par de papilas en la base de la parte interna del tubo de la corola en $S$. fulgens y su ausencia en $S$. gesneriiflora, y c) longitud de la porción atenuada del ápice de las brácteas florales, en S. gesneriiflora va de 5.1 a $7.0 \mathrm{~mm}$ mientras en $S$. fulgens de 9.0 a $19.6 \mathrm{~mm}$. Cabe señalar que las dos primeras características ya habían sido utilizadas por Ramamoorthy (2005) para distinguirlas.

La distribución de Salvia fulgens está embebida dentro de la de S. gesneriiflora (Fig. 2), aunque la anterior además extiende su área más hacia el norte en los estados de Colima, Durango, Jalisco, Nayarit, Sinaloa y Zacatecas y ocupa espacios en la porción sur de la Sierra Madre Occidental, aparte de los ya mencionados en la Franja Volcánica Transmexicana (Fig. 2). 

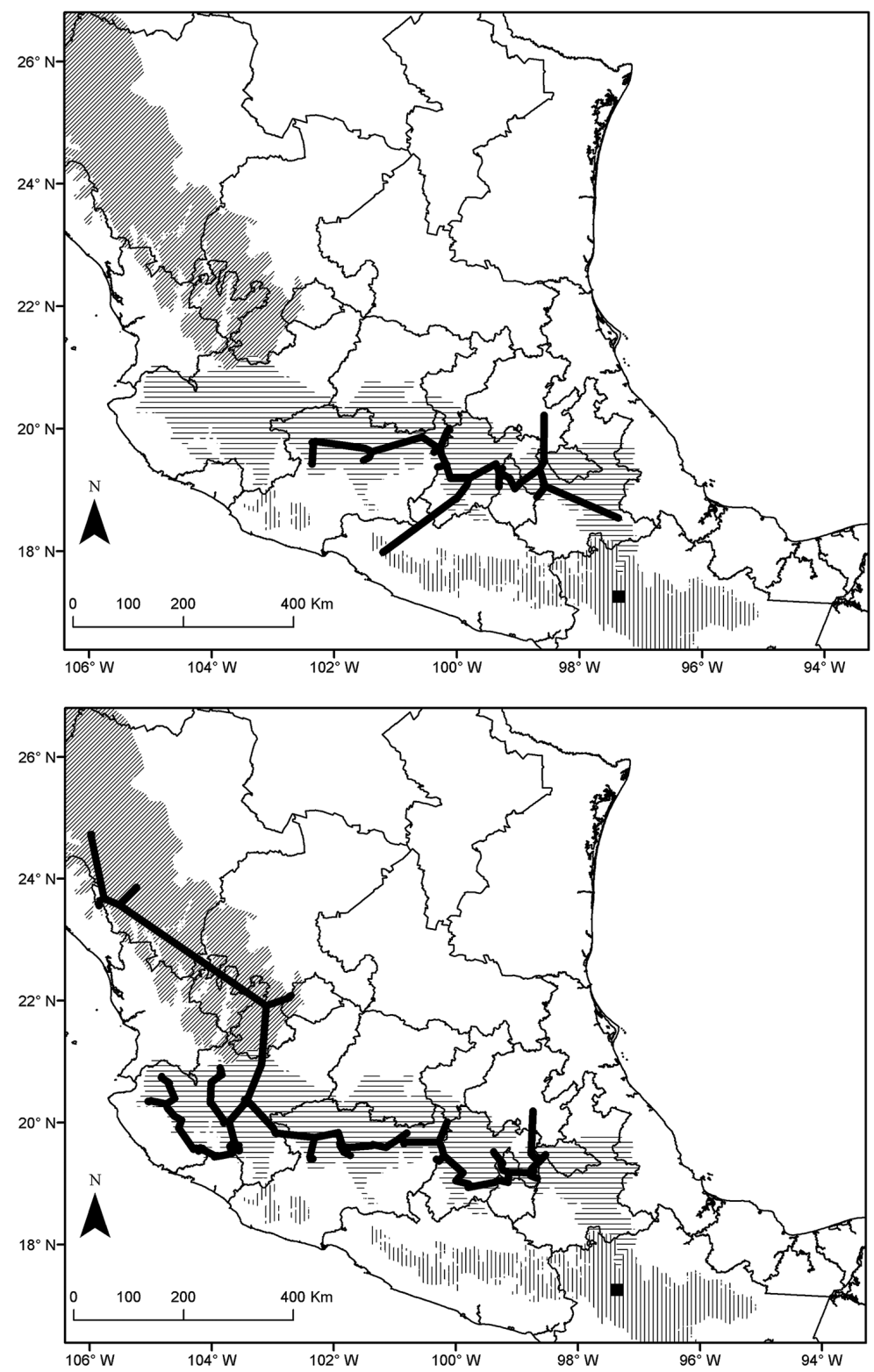

Fig. 2. Mapa de distribución mediante trazos individuales de Salvia fulgens (porción superior) y $S$. gesneriiflora (porción inferior). Salvia tilantongensis sp. nov. se conoce de una sola localidad y está señalada con un cuadrado. Las áreas de la Sierra Madre del Sur (sombreado de líneas verticales), la Faja Volcánica Transmexicana (sombreado de líneas horizontales) y la Sierra Madre Occidental (sombreado de líneas diagonales) están resaltadas como referencia. 
Salvia gesneriiflora Lindl. \& Paxton, Paxton's Fl. Gard. 2: 49, t. 47. 1851. Tipo: lámina 47, Lindley \& Paxton, 1851; elaborada con base en un ejemplar vivo cultivado a partir de semillas colectadas en México. Fig. 1B.

Salvia fulgens fo. gesneriiflora (Lindl. \& Paxton) Voss, Vilm. Blumengärtn. (ed. 3) 1: 840. 1895.

Arbusto 0.8 a $2.5(-4) \mathrm{m}$ de alto, a veces un poco decumbente o escandente; tallos tomentosos a pilosos y puberulentos, por lo general cubiertos con abundantes tricomas glandular-capitados. Hojas con peciolo 1.3 a $4.6(-8.0) \mathrm{cm}$ de largo, piloso y cubierto por tricomas glandular-capitados cortos y glándulas sésiles; lámina ampliamente ovada a ovado-lanceolada, (3.0-)5.3 a 10.3(-16.0) $\times 2.0$ a $6.6(-10.9) \mathrm{cm}$, aguda a acuminada o atenuada hacia el ápice, cordada a truncada o en ocasiones redondeada en la base, serrulada en el margen, pilosa o con tricomas adpresos en el haz, por lo común blanco-tomentosa y a menudo con puntos glandulares en el envés, las hojas jóvenes con tricomas glandular-capitados en ambas caras. Inflorescencia terminal, de 10 a 30(-44) cm de largo, 4 a 11(-17) verticilastros, cada uno con 2 a 6(-10) flores, los de la base de la inflorescencia separados entre sí por 1.4 a $3.0(-4.0) \mathrm{cm}$, eje floral piloso y cubierto con tricomas glandular-capitados. Bráctea floral ovada a ovadolanceolada, (0.6-) 0.7 a $2.0(-2.4) \times 0.2$ a $0.7(-1.0) \mathrm{cm}$, decidua, verde o roja, truncada en la base, atenuada en el ápice ((0.2-)1 a $3.2 \mathrm{~mm})$, margen entero, pilosa en la cara externa y con tricomas glandular-capitados. Flores con pedicelos 5 a $10 \mathrm{~mm}$ de largo (hasta $12 \mathrm{~mm}$ en fruto), pilosos y con tricomas glandular-capitados. Cáliz (1.3-)1.7 a $2.5(-3.0) \mathrm{cm}$ de largo, 0.5 a $0.8(-1.0) \mathrm{cm}$ de ancho en la garganta, verde o rojo, piloso a hispídulo en el exterior y con tricomas glandular-capitados, hispídulo a glabro en el interior y hacia los labios, lóbulos agudos, el superior entero y con 5 o 7 venas. Corola de color rojo brillante, pilosa en el labio superior y en el dorso de la porción media distal, o en algunas ocasiones con algunos tricomas dispersos a todo lo largo; tubo 3.2 a $4.0(-4.4) \mathrm{cm}$ de largo, por lo general ventricoso, 0.6 a $1.0(-1.2) \mathrm{cm}$ de ancho en el vientre, recto en la base y epapilado; labio superior 1.6 a $2.1(-2.5) \mathrm{cm}$ de largo, labio inferior 1.2 a $2.0(-2.4) \times 0.5$ a $1.0 \mathrm{~cm}$, lóbulo medio reflejo. Estambres insertos en la corola; filamento 0.6 a $0.8(-0.9) \mathrm{cm}$ de largo; conectivo (1.9-)2.0 a $2.6 \mathrm{~cm}$ de largo, con un diente corto y retrorso después de la inserción del filamento hacia la porción posterior, o bien sin ornamentaciones, puberulento en la cara dorsal y ventral en torno a la inserción del filamento; teca 3.6 a $4.5(-5.0) \mathrm{mm}$ de largo; un par de estaminodios filiformes presentes atrás y arriba de la inserción del filamento a la corola. Cuerno ginobásico 1.0 a 1.6(-1.9) $\mathrm{mm}$ de largo; estilo 4.7 a $5.9(-6.6) \mathrm{cm}$ de largo, piloso hacia el ápice, en las caras dorsal y ventral, rama superior arqueada, (5-) 7 a $8 \mathrm{~mm}$ de largo, inferior aguda y recta, 2.0 a $3.2 \mathrm{~mm}$ de largo. Mericarpio ovoide, 
2.8 a $3.5 \times 1.6$ a $2.0 \mathrm{~mm}$, café oscuro uniforme o café claro y jaspeado con un tono más oscuro, liso y glabro.

Material selecto examinado: MÉXICO, Aguascalientes. Calvillo: Salto del Pilar, 2450 m, 15 abr 1985, M. de La Cerda-L. s.n. (HUAA). Colima. Comala: Parque Nacional Nevado de Colima, camino Comala - San Antonio - Nuevo Pueblo San Antonio, 19²9'20" N 103³9'57" W, 1770 m, 14 abr 2001, G. Ibarra-M. et al. 4861 (MEXU); Minatitlán: Sierra of Manantlán, road from el Sauz to El Terrero, 19²6'51.6" N 10356'6.47" W, 922 m, 5 dic 1999, J. Cahill \& M. J. Cházaro-B.3024 (CIMI, HUMO, MEXU, MICH). Distrito Federal. Magdalena Contreras: Rancho Pachita, 2700 m, 27 mar 1977, A. Ventura-A. 2637 (CIIDIR, IBUG, MEXU); Capultitla San Bernabé, 2750 m, 13 mar 1977, A. Ventura-A. 2639 (MEXU). Durango. Otaez: $5 \mathrm{~km}$ de Otaez, sobre el camino a Los Altares, $2300 \mathrm{~m}, 4$ oct 1990, A. Benitez-P. 2621 (CIIDIR, MEXU); Pueblo Nuevo: 8 km al E de El Palmito, carretera Mazatlán - Durango, 10 mar 1985, P. Tenorio-L. \& C. Romero de T. 8238 (MEXU). Estado de México. Amecameca: cerro Sacromonte, 2100 m, 2 nov 1981, J. A. Vázquez-G. 637 (IBUG); 1 km al NE de Santo Tomás de Atzingo, 1450 m, L. Pérez-J. 204 (IEB, OAX); Chalco: cerca de la colonia Agrícola Manuel Ávila Camacho, $2750 \mathrm{~m}, 11$ sep 1966, A. Vargas-N. 2750 (IBUG); Coatepec de Harinas: El Salto, 1856'14.9" N 99 47'55.2" W, 2239 m, 28 feb 2010, N. Muñoz-C. 379 (MEXU); Donato Guerra: cerro Pelón, $19^{\circ} 21^{\prime} 23^{\prime \prime} \mathrm{N} 1001^{\circ} 16^{\prime} 18^{\prime \prime} \mathrm{W}, 2300 \mathrm{~m}, 2$ may 2005, J. Martínez-C. et al. 1646 (IEB, MEXU); Jilotzingo: 3 km al NO de San Luis Ayucan, 2850 m, 29 ene 1978, R. López-G. 11756 (GUADA); Ocuilan: San Juan Atzingo, 19¹' N 99²2' W, 2600 m, 6 feb 1987, J. Castañeda-R. \& P. Trejo-G. 48 (MEXU); Temascaltepec: Mesón Viejo, NE of Temascaltepec, on highway 130, 99 $54^{\circ}$ N 19 $9^{\circ} 9^{\prime} \mathrm{W}, 2800 \mathrm{~m}$, 10 nov 1988, S. A. Reisfield 1257 (MEXU); Texcoco: Sta. Catarina, 2300 m, 26 oct 1985, C. García s.n. (IBUG); Tlalmanalco: 3 km al E de San Rafael, 2800 m, 1 ene 1965, J. Rzedowski 19339 (MEXU); San Rafael, 2500 m, 19 feb 1983, E. Ventura-V. 550 (IEB, OAX). Hidalgo. Pachuca: cerro de Las Ventanas, $6 \mathrm{~km}$ al $\mathrm{N}$ de Pachuca, 2900 m, 25 jun 1967, C. Jiménez-R. 94-A (MEXU). Jalisco. Ahualulco del Mercado: Ahualulco, camino al cerro Bola, aprox. $10 \mathrm{~km}$ al SE, $1600 \mathrm{~m}, 30$ ene 1983, A. de Santiago-C. 2 (IBUG); Autlán de Navarro: Sierra de Cacoma, $400 \mathrm{~m}$ al S del Punto de Santa Mónica, 2100 m, 7 ene 1995, O. Vargas-P. \& R. González-T. s.n. (IBUG); Ciudad Guzmán: km 14 brecha El Fresnito-Antenas del Canal 13, Nevado de Colima, 2660 m, 12 sep 1983, R. Ramírez-D. \& L. M. González-V. 518 (IBUG); Cuautitlán: brecha a Las Capillas, Sierra de Manantlán, 2750 m, 20 nov 1981, L. M. Villarreal de Puga et al. 11974 (IBUG); Jocotepec: cerro Grande, al N de Jocotepec, Sierra del 
Madroño, 2400 m, 26 ene 1983, O. Reyna-B. 89 (IBUG); Mazamitla: 3 km al SW de Mazamitla, 1800 m, 16 dic 1981, J. J. Velasco-R. s.n. (IBUG); Puerto Vallarta: rocky crest of low mountains of the Pacific slope, $7 \mathrm{~km}$ by $\mathrm{rd}$. E of the crossing (El Crucero) Unidad Cuale-Talpa (a little pass with rd. jct. on top of Sierra El Cuale), $6 \mathrm{~km}$ due SE of Cuale and due E of the Minas de Zimapan, 2250-2340 m, 18 ago 1993, T. S. Cochrane et al. 13141 (IBUG); San Martín Hidalgo: cerro El Huehuentón, al SW de Lagunillas, 2400 m, 2 ene 1987, L. M. González-V. 2870 (IBUG); San Sebastián del Oeste: La Bufa, 7 abr 1993, J. J. Reynoso-D. et al. 1262 (IBUG); Talpa de Allende: La Torre, $8 \mathrm{~km}$ al W de Cuale, $2200 \mathrm{~m}, 22$ ene 2000, L. M. González-V. 4648 (IBUG); Tecolotlán: sierra de Quila, ladera SE del cerro Huehuentón, 2100 m, 4 feb 1990, J. J. Guerrero-N. 661 (IBUG); Tequila: cerro de Tequila, 1200-2500 m, 26 feb 1967, L. M. Villarreal de Puga 12543 (IBUG); Tolimán: en las faldas del Nevado de Colima, 2000 m, 25 nov 1985, C. O. Nathal-E. 45 (IBUG); Tonila: brecha a las Joyas, Nevado de Colima, 2340 m, 9 feb 1986, R. Ramirez-D. 215 (IBUG, IEB); Tuxpan: Nevado de Colima, 2600 m, 10 feb 1974, L. M. Villarreal de Puga 6048 (IBUG); Zapopan: $40 \mathrm{~km}$ de la carretera a Saltillo, 20 abr 1978, J. Rivera \& Quevedo s.n. (IBUG); Zapotitlán de Vadillo: $10 \mathrm{~km}$ sobre la desviación al Nevado de Colima, a partir de El Fresnito, 2510 m, 6 abr 1988, A. G. Mendoza 3826 (IBUG, IEB). Michoacán. Angangueo: Chincua, terrenos federales, 19²40'28" N 100¹6'36" W, 3217 m, 27 oct 2004, J. Martínez-C. et al. 1314 (MEXU); Charo: cerro de la Espadilla cerca de Las Mesas, 2400 m, 8 may 1987, J. S. Martínez 2065 (CIIDIR, IBUG, IEB); Cherán: cerro de La Virgen, 3100 m, 15 oct 1990, E. García \& E. Pérez 3328 (IBUG); Contepec: 2 km de Santa María la Ahogada, 200'37" N 1008'52" W, 2420 m, 23 ago 2004, M. G. Cornejo-T. et al. 852 (MEXU); Erongarícuaro: cerro Las Varas, 2300 m, 7 nov 1985, H. Díaz-B.1694 (GUADA, IEB, OAX); Hidalgo: a 3 $\mathrm{km}$ al S de Mil Cumbres, $2200 \mathrm{~m}, 15$ may 1985, J. C. Soto-N. et al. 8520 (CIIDIR); Indaparapeo: cerro El Acre, cerca del rancho Rosa Jorio, 2400 m, 18 mar 1986, J. S. Martínez 1257 (CIIDIR, IEB); Morelia: cerro Pico Azul, cerca de Jesús del Monte, 2500 m, 2 may 1985, J. S. Martínez 3928 (MEXU); Nahuatzen: cerro La Bandera, cerca de Comachuen, municipios de Nahuatzen y Tingambato, $2850 \mathrm{~m}$, 6 feb 1986, A. Martínez-L. 758 (CIIDIR, IBUG, IEB); Ocampo: ejido El Rosario, límite del Santuario de la Mariposa Monarca, 2000 m, 25 feb 1989, A. Flores-H. et al. 1506 (IBUG); Pátzcuaro: cerro El Burro, 2400 m, 2 dic 1985, J. M. Escobedo 748 (CIIDIR, IBUG, IEB); Queréndaro: Cañada Real, cerca de San José de la Cumbre, 2600 m, 12 abr 1986, J. S. Martínez 1233 (CIIDIR, IBUG), Ibid. 1295 (OAX); Salvador Escalante: cerro La Tapada, ejido Felipe Tzinzun, 3075 m, 21 ene 1988, S. Zamudio 6029 (CIIDIR, GUADA, IBUG, IEB); Zirahuén, 2100 m, 1 mar 1989, E. 
Pérez-C. 472 (IEB, MEXU); Tancítaro: cerro Tancítaro, $27 \mathrm{~km}$ al W en línea recta, $3 \mathrm{~km}$ al E de Apo, camino a Parastaco, 19²6'15" N 102 $23^{\circ} 35^{\prime \prime} \mathrm{W}, 2280 \mathrm{~m}, 11 \mathrm{mar}$ 1998, I. García-R. 5061 (CIMI, IEB, MICH); Tingambato: barranca al NW y NE de Tingambato, 2000 m, 22 abr 1995, M. Cruz-E. 44 (MEXU); Zacapu: $1.5 \mathrm{~km}$ al N de El Pueblito, 4 nov 1985, A. Martínez-L. 97 (IBUG, IEB); Zinapécuaro: cañada El Salto, cerca de Bocaneo, 2000 m, 1 mar 1988, J. S. Martínez 1230 (CIIDIR, IBUG, OAX); Zitácuaro: zona 7, ladera NW del cerro Cacique, 19²3'50" N 100¹9'7" W, 2340 m, 4 mar 1979, G. Ibarra-C. 384 (MEXU). Morelos. Huitzilac: Rancho San Lorenzo, km 53.5 de la carretera federal México - Acapulco 95, al SW del poblado Tres Marías, 19³9' N 99²14' W, 2650 m, 7 sep 1989, I. Díaz-V. \& R. Noriega-T. 1154 (MEXU). Puebla. El Salto, Río Frío, 3000 m, 10 abr 1974, L. W. Boege 3079 (MEXU). Sinaloa. Concordia: 2-4 km SW of El Palmito, 1980 m, 7 abr 1988, D. E. Breedlove \& B. Bartholomew 66486 (MEXU). Tlaxcala. Nanacamilpa: Nanacamilpa, $2810 \mathrm{~m}$, 21 oct 1985, F. A. Pérez-M. s.n. (IBUG). Zacatecas. El Plateado de Joaquín Amaro: barranca de Las Mujeres, El Plateado, 2400 m, mar 1964, L. Vela-G. 1475 (MEXU).

Salvia gesneriiflora crece en la Sierra Madre Occidental y en la Franja Volcánica Transmexicana (Fig. 2). Está presente en el Distrito Federal y en los estados de Aguascalientes, Colima, Durango, Estado de México, Hidalgo, Jalisco, México, Michoacán, Morelos, Puebla, Sinaloa, Tlaxcala y Zacatecas (Fig. 2); también se le registra de Guerrero (Epling, 1939) y de Nayarit (Téllez, 1995), aunque no se observaron especímenes de herbario que así lo atestigüen. Habita en bosques de pino y encino, pino, encino, oyamel, y mesófilo de montaña. Es frecuente encontrar poblaciones densas a la orilla de brechas y claros dentro del bosque. El rango altitudinal que ocupa va de (1500-)1900 a 2800(-3600) m. La floración y fructificación son sincrónicas y se desarrollan durante todo el año, con una mayor presencia de octubre a marzo. En Zinapécuaro, Michoacán, suelen conocer a la especie como retama de monte.

Salvia tilantongensis J.G. González \& R. Aguilar-Santelises sp. nov. Tipo: MÉXICO. Oaxaca, Dto. Nochixtlán, Santiago Tilantongo, $6 \mathrm{~km}$ al O de Santiago Tilan-

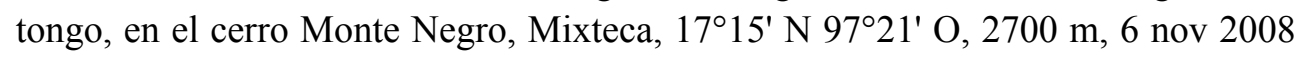
(fl, fr), R. Martínez-García 84 (holotipo OAX, isotipos ENCB, IBUG, IEB, MEXU, SERO). Fig. $1 \mathrm{C}$ y D, y 3.

Salvia tilantongensis ad sectionem Nobiles Epling pertinet sed ab aliis speciebus huius sectionis differt propter caules desquamatos, petiolos articulatos, folia potius 
angusta, bracteas florales parvas (minus quam $8 \mathrm{~mm}$ longas), fructus cum pedicellis longis (usque ad $17.8 \mathrm{~mm}$ ), et lobulos calycis longe aristatos (aristis 3-4.5 mm longis).

Arbusto 1.0 a 2.0(-2.5) $\mathrm{m}$ de alto, erecto; tallo piloso y con tricomas glandularcapitados, estos últimos concentrados hacia la inflorescencia, peciolos y ramas jóvenes, corteza exfoliante en láminas longitudinales en las ramas principales. Hojas con peciolo (1.2-)1.8 a 2.9(-3.5) cm de largo, articulado en la base sobre una prolongación ensanchada del entrenudo, densamente piloso y con tricomas glandular-capitados; lámina lanceolada a ovado-lanceolada, 5.2 a $8.0(-10.0) \times 1.5$ a $4.5(-5.1) \mathrm{cm}$, aguda hacia el ápice, cordada y cortamente cuneada en la base, crenada a ligeramente serrada en el margen, cubierta con tricomas adpresos dispersos en el haz, moderada a densamente pilosa en el envés. Inflorescencia terminal, de 5.3 a 11.2(-15.0) $\mathrm{cm}$ de largo, (4-)6 a 12 verticilastros, cada uno con 2 a 4(-6) flores, los de la base de la inflorescencia separados entre sí 0.6 a $1.5(-2.2) \mathrm{cm}$, eje floral glabro o con tricomas glandular-capitados. Bráctea floral ovada, 0.2 a $0.5(-0.8) \times 0.2$ a $0.4(-0.5)$ $\mathrm{cm}$, decidua, verde o roja, acuminada en el ápice (porción atenuada 5.1 a $7.0 \mathrm{~mm}$ de largo), truncada en la base, margen entero, pilosa y cubierta por tricomas glandularcapitados en la cara externa, la interna glabra. Flores con pedicelos 0.5 a $1.0(-1.1) \mathrm{cm}$ de largo en flor, hasta $17.8 \mathrm{~mm}$ de largo en fruto, densamente cubierto por tricomas glandular-capitados de 0.1 a $0.2 \mathrm{~mm}$ de largo. Cáliz (1.3-)1.5 a 2.0(-2.3) cm de largo, 0.7 a $0.9 \mathrm{~cm}$ de ancho en la garganta (hasta $2.5 \times 1.1 \mathrm{~cm}$ en fruto), rojo (pajizo al secarse), esparcidamente cubierto por tricomas glandular-capitados concentrados sobre todo en las venas, en el interior cubierto por tricomas adpresos o verrucoso, lóbulos agudos y rematados por una arista de 3.0 a $4.5 \mathrm{~mm}$ de largo, el superior entero y con 7 venas. Corola de color rojo brillante, esparcidamente pilosa hacia los labios; tubo de 3.8-4.3 cm de largo, ligeramente ventricoso, (0.5-)0.6 a $1.0 \mathrm{~cm}$ de ancho en el vientre, recto en la base y sin papilas en el interior; labio superior 1.6 a $2.4 \mathrm{~cm}$ de largo, labio inferior (1.2-)1.7 a $2.7 \times 1.0$ a $1.5 \mathrm{~cm}$, con el lóbulo medio incurvado cóncavo. Estambres insertos en la corola; filamentos 4 a $6 \mathrm{~mm}$ de largo; conectivos 1.9 a $2.4 \mathrm{~cm}$ de largo, puberulentos en el dorso y pilosos junto a la inserción del filamento; tecas 2.4 a $3.3 \mathrm{~mm}$ de largo; estaminodios representados por un par de papilas arqueadas de 0.4 a $0.5 \mathrm{~mm}$ de largo en la porción media del tubo, atrás y arriba de la inserción del filamento. Cuerno de la ginobase 0.2 a $1.0 \mathrm{~mm}$ de largo; estilos 4.3 a $4.5 \mathrm{~cm}$ de largo, densamente pilosos en el tercio apical; rama superior (5.4-)6.4 a $8.0(-8.8) \mathrm{mm}$ de largo, la inferior 5.3 a 6.1(-7.0) $\mathrm{mm}$ de largo. Mericarpio ovoide, 2.8 a $3.2 \times 1.6$ a $2.2 \mathrm{~mm}$, café amarillento a veces jaspeado de manera irregular con un tono más oscuro, liso y glabro. 


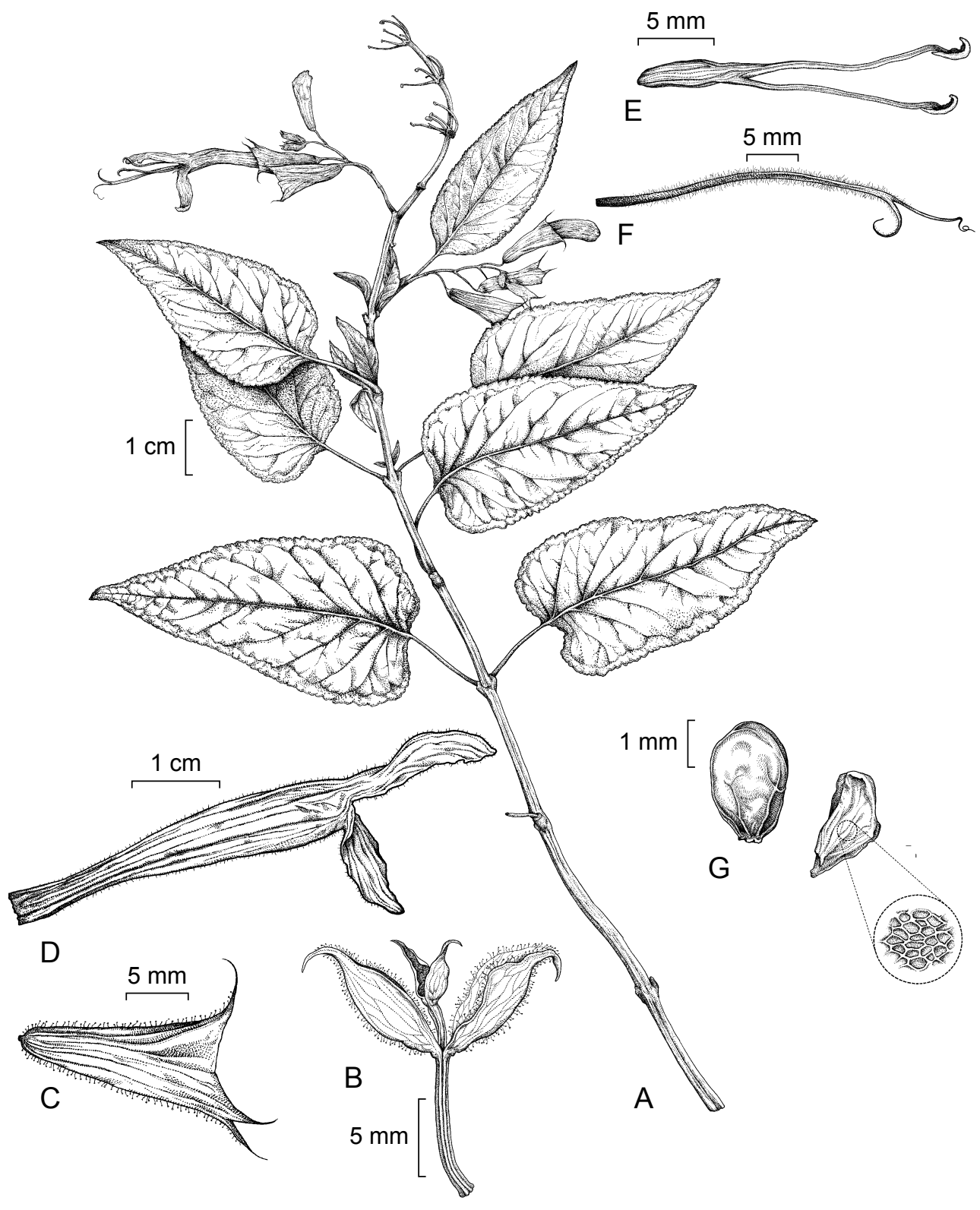

Fig. 3. Salvia tilantongensis sp. nov. A. Aspecto general de la planta mostrado en una rama; B. brácteas florales en la porción apical de una inflorescencia; C. cáliz; D. corola; E. conectivos y tecas; F. porción apical del estilo; G. mericarpio maduro (izquierda) y semilla (derecha), con un acercamiento de su superficie (ilustración basada en R. Martínez-García 84 y elaborada por Daniel Acosta-Aguilar). 
Material adicional examinado: MÉXICO. Oaxaca, Santiago Tilantongo: brecha hacia las ruinas arqueológicas de Santiago Tilantongo, $1 \mathrm{~km}$ en línea recta al SO

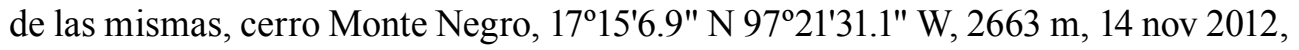
J. G. González-Gallegos \& G. Santos 1400 (IBUG).

Salvia tilantongensis es una especie endémica restringida a la Mixteca Alta en el Estado de Oaxaca, México. Ocupa un intervalo de elevación aproximado de 2600 a $2800 \mathrm{~m}$. Crece en bosques de encino bien conservados u ocasionalmente en zonas con cierto grado de disturbio. Comparte hábitat con Quercus laurina Bonpl. Florece y fructifica de octubre a diciembre.

Las características del nuevo taxon lo relacionan directamente con la sección Nobiles Epling. Ésta incluye arbustos o hierbas perennes, de hojas ampliamente ovadas o elíptico-lanceoladas, glabras o moderadamente vellosas, con brácteas florales deciduas o persistentes, inflorescencias en racimos o en ocasiones con flores dispuestas en las axilas de las hojas hacia el ápice de las ramas, labio superior de los cálices con 5 a 7 venas, corola roja con el tubo ventricoso o ampliado hacia el ápice, desnudo en el interior (sin papilas), labio inferior tan largo como el superior o más corto, estambres inclusos, conectivo entero o, especialmente en las especies mexicanas, con un diente pequeño posterior a la inserción con el filamento, estilo velloso en el dorso y vientre. Se propone al taxon nuevo como parte de esta sección de manera provisional ya que la evidencia filogenética disponible no respalda la monofilia del grupo ni de la mayoría de las secciones propuestas por Epling (Jenks et al., 2013), pero es claro que en el futuro cercano será necesaria la reestructuración de la clasificación infragenérica en su totalidad. Esta situación ya había sido anticipada por Dos Santos (1991), quien recircunscribe a Nobiles al excluir a las especies mexicanas, y por Ramamoorthy (1984) quien transfiere a S. cardinalis (sección Cardinalis Epling (1939)) como sinónimo de S. fulgens y erige la sección Holwayana Ramamoorthy para albergar al remanente de especies de la sección Cardinalis. Sin embargo, en el presente trabajo se prefiere el sistema propuesto por Epling (1939). Entre las especies de dicha sección, $S$. tilantongensis es muy similar en su morfología a $S$. gesneriiflora. Difiere de ella debido a sus tallos exfoliantes, peciolos articulados en un ensanchamiento del nudo a manera de base, láminas foliares por lo regular más estrechas, inflorescencias que no superan los $15 \mathrm{~cm}$ de largo, brácteas florales más cortas y de ápice reducido, pedicelos más largos durante la fructificación, lóbulos del cáliz largamente aristados, el lóbulo medio del labio inferior de la corola incurvado-cóncavo, filamento más corto, estilo más corto y rama inferior del estilo más larga (Cuadro 1). Salvia fulgens de la sección Fulgentes Epling también es 
Cuadro 1. Comparación de características morfológicas distintivas, distribución, intervalo altitudinal y hábitat entre Salvia tilantongensis y especies morfológicamente semejantes.

\begin{tabular}{|c|c|c|c|}
\hline Características & S. fulgens & S. gesneriiflora & S. tilantongensis \\
\hline \multicolumn{4}{|l|}{ TALLO } \\
\hline Exfoliante & no & no & sí \\
\hline \multicolumn{4}{|l|}{ HOJA } \\
\hline Inserción del peciolo & directa al tallo & directa al tallo & $\begin{array}{l}\text { sobre una } \\
\text { prolongación } \\
\text { engrosada del } \\
\text { entrenudo }\end{array}$ \\
\hline \multicolumn{4}{|l|}{ INFLORESCENCIA } \\
\hline $\begin{array}{l}\text { Longitud del racimo } \\
(\mathrm{cm})\end{array}$ & 9.5 a 30 & 10 a $30(-44)$ & 5.3 a $11.2(-15.0)$ \\
\hline \multicolumn{4}{|l|}{$\begin{array}{l}\text { BRÁCTEA } \\
\text { FLORAL }\end{array}$} \\
\hline Tamaño (cm) & $\begin{array}{l}(1.9-) 2.0 \text { a } 3.6(-4.3) \\
\times 0.6 \text { a } 1.0\end{array}$ & $\begin{array}{l}(0.6-) 0.7 \text { a } 2.0(-2.4) \times \\
0.2 \text { a } 0.7(-1.0)\end{array}$ & $\begin{array}{l}0.2 \text { a } 0.5(-0.8) \times \\
0.2 \text { a } 0.4(-0.5)\end{array}$ \\
\hline $\begin{array}{l}\text { Longitud de la } \\
\text { porción atenuada del } \\
\text { ápice }(\mathrm{mm})\end{array}$ & 9.0 a 19.6 & $(0.2-) 1.0$ a 3.2 & 5.1 a 7.0 \\
\hline \multicolumn{4}{|l|}{ CÁLIZ } \\
\hline Forma de los lóbulos & $\begin{array}{l}\text { agudos y no } \\
\text { largamente aristados }\end{array}$ & $\begin{array}{l}\text { agudos y no } \\
\text { largamente aristados }\end{array}$ & $\begin{array}{l}\text { agudos y } \\
\text { largamente } \\
\text { aristados (aristas } \\
3.0 \text { a } 4.5 \mathrm{~mm} \text { de } \\
\text { largo) }\end{array}$ \\
\hline \multicolumn{4}{|l|}{ COROLA } \\
\hline $\begin{array}{l}\text { Forma del lóbulo } \\
\text { medio del labio } \\
\text { inferior }\end{array}$ & deflexo & deflexo & incurvado cóncavo \\
\hline $\begin{array}{l}\text { Número de papilas } \\
\text { en la porción interna } \\
\text { basal del tubo }\end{array}$ & 2 & 0 & 0 \\
\hline \multicolumn{4}{|l|}{ GINECEO } \\
\hline $\begin{array}{l}\text { Longitud del estilo } \\
(\mathrm{cm})\end{array}$ & 4.0 a $5.3(-5.6)$ & 4.7 a $5.9(-6.6)$ & 4.3 a 4.5 \\
\hline $\begin{array}{l}\text { Longitud de la rama } \\
\text { superior }(\mathrm{mm})\end{array}$ & $(2.5-) 3.9$ a 8.9 & $(5-) 7$ a 8 & $\begin{array}{l}(5.4-) 6.4 \text { a } 8.0 \\
(-8.8)\end{array}$ \\
\hline
\end{tabular}


Cuadro 1. Continuación.

\begin{tabular}{|c|c|c|c|}
\hline Características & S. fulgens & S. gesneriiflora & S. tilantongensis \\
\hline $\begin{array}{l}\text { Longitud de la rama } \\
\text { inferior }(\mathrm{mm})\end{array}$ & $(1.3-) 2.1$ a 3.7 & 2.0 a 3.2 & 5.3 a $6.1(-7.0)$ \\
\hline $\begin{array}{l}\text { Distribución } \\
\text { geográfica }\end{array}$ & $\begin{array}{l}\text { Distrito Federal, } \\
\text { Estado de México, } \\
\text { Guerrero, Hidalgo, } \\
\text { Michoacán, } \\
\text { Morelos, Puebla y } \\
\text { Tlaxcala }\end{array}$ & $\begin{array}{l}\text { Aguascalientes, } \\
\text { Colima, Durango, } \\
\text { Estado de México, } \\
\text { Hidalgo, Jalisco, } \\
\text { Michoacán, Morelos, } \\
\text { Puebla, Sinaloa, } \\
\text { Tlaxcala y Zacatecas }\end{array}$ & Oaxaca \\
\hline $\begin{array}{l}\text { Intervalo altitudinal } \\
\text { (m) }\end{array}$ & 2400 a $3400(-3990)$ & $\begin{array}{l}(1500-) 1900 \text { a } 2800 \\
(-3600) .\end{array}$ & 2600 a 2800 \\
\hline Hábitat & $\begin{array}{l}\text { bosque de pino, } \\
\text { encino, mesófilo } \\
\text { de montaña, de } \\
\text { oyamel y rara vez en } \\
\text { matorral xerófilo }\end{array}$ & $\begin{array}{l}\text { bosque de pino- } \\
\text { encino, encino, pino, } \\
\text { oyamel y mesófilo de } \\
\text { montaña }\end{array}$ & bosque de encino \\
\hline
\end{tabular}

semejante en su morfología al nuevo taxon. Sin embargo, se distingue claramente de S. gesneriiflora y $S$. tilantongensis por la presencia de un par de papilas en la parte basal e interna del tubo de la corola, los pedicelos con tricomas glandular-capitados de menos de $0.05 \mathrm{~mm}$ de largo, las brácteas florales por lo usual más largas y con una atenuación más pronunciada en el ápice de la bráctea (Cuadro 1). Es de notar que el engrosamiento del punto de inserción del peciolo con el nudo en el tallo ya había sido utilizado como diagnóstico por Bedolla-García et al. (2011) y que es recomendable un análisis de su distribución y valor taxonómico dentro del género.

El nombre de la especie hace referencia al municipio en que esta planta ha sido colectada hasta el momento: Santiago Tilantongo, Oaxaca, México.

Clave para la determinación de Salvia tilantongensis y especies morfológicamente afines

1a Brácteas florales de (1.9-)2.0 a 3.6(-4.3) cm de largo, porción atenuada del ápice prolongada 9.0 a $19.6 \mathrm{~mm}$; pedicelos con tricomas glandular-capitados de me- 
nos de $0.05 \mathrm{~mm}$ de largo; un par de papilas presentes en la parte interna y basal del tubo de la corola S. fulgens $1 \mathrm{~b}$ Brácteas florales por lo general menores de $20 \mathrm{~mm}$ de largo, con la porción apical menor de $8 \mathrm{~mm}$; pedicelos con tricomas glandular-capitados de 0.1-0.2 mm de largo; el tubo de la corola sin papilas en el interior hacia su base

2a Tallo con corteza no exfoliante; peciolo articulado directamente al tallo; láminas foliares hasta de $10.9 \mathrm{~cm}$ de ancho; inflorescencias de hasta de $44 \mathrm{~cm}$ de largo; brácteas florales de (0.6-)0.7 a 2.0(-2.4) mm de largo; pedicelos en fruto no más de $12 \mathrm{~mm}$ de largo; lóbulos del cáliz no aristados; lóbulo medio del labio inferior de la corola reflejo; filamento de 6 a 8(-9) $\mathrm{mm}$ de largo; estilo de 4.7 a $5.9(-6.6) \mathrm{cm}$ de largo, rama inferior 2.0 a $3.2 \mathrm{~mm}$ de largo. Plantas distribuidas por las serranías del Pacífico desde Durango hasta Guerrero y luego penetrando por el centro del país hasta Hidalgo, Puebla y Tlaxcala ....

S. gesneriiflora

2b Tallo con corteza exfoliante; peciolo articulado a una base engrosada a partir del nudo; láminas foliares sin sobrepasar $5.1 \mathrm{~cm}$ de ancho; inflorescencias hasta de $15 \mathrm{~cm}$ de largo; brácteas florales de 0.2 a $0.5(-0.8) \mathrm{mm}$ de largo; pedicelos en fruto hasta de $17.8 \mathrm{~mm}$ de largo; lóbulos del cáliz con una arista de 3.0 a $4.5 \mathrm{~mm}$ de largo; lóbulo medio del labio inferior de la corola incurvado-cóncavo, de tal manera que forma una capucha; filamento 4 a $6 \mathrm{~mm}$ de largo; estilo de 4.3 a $4.5 \mathrm{~cm}$ de largo, rama inferior 5.3 a $6.1(-7.0) \mathrm{mm}$ de largo. Plantas exclusivas de Oaxaca

S. tilantongensis

El traslape de la variación de la mayoría de las características morfológicas y de gran parte de la distribución geográfica y tipo de hábitat ocupado entre Salvia fulgens y $S$. gesneriiflora hacen difícil su distinción y ha llevado en ocasiones a pensar que pudiera tratarse de taxones conespecíficos (Ramamoorthy, 1985). En el presente trabajo se decide reconocerlas como entidades distintas con base en tres características morfológicas: las longitudes de la bráctea floral y de la porción atenuada de su ápice, y la presencia o ausencia de papilas en el interior del tubo de la corola. Sin embargo, estudios genéticos y filogeográficos podrían esclarecer de mejor manera esta situación. Además, si se trata de especies diferentes que conviven en la misma área geográfica y tipo de hábitat, entre las que no existen diferencias florales abruptas, sería interesante analizar qué factores evitan el flujo génico entre ellas y las mantienen como grupos cohesivos. En contraste, Salvia tilantongensis muestra una morfología más distintiva respecto a cualquiera de los taxa anteriores y exhibe una propiedad inusual en el género: sus tallos exfoliantes. 


\section{AGRADECIMIENTOS}

Agradecemos a Remedios Martínez-García quien realizó la primer colecta de Salvia tilantongensis y nos proveyó información sobre la misma. Dirigimos un especial reconocimiento a las autoridades de Santiago Tilantongo por la hospitalidad y facilidades brindadas, en especial a Gervasio Santos por su valiosa colaboración durante el desarrollo del trabajo de campo. La diagnosis en latín fue elaborada por el especialista Juan Acosta-Aguilar, y la ilustración por el artista gráfico Daniel Acosta-Aguilar. María Elena Mendoza López nos proporcionó datos sobre sus colectas de S. gesneriiflora en Aguascalientes. Los revisores de este documento y el comité editorial de la revista ayudaron a mejorar el trabajo con sus observaciones críticas. El soporte financiero fue otorgado de manera parcial por el Instituto Politécnico Nacional (proyectos CGPI 20050340 y SIP 20121168), la Universidad de Guadalajara, el Consejo Nacional de Ciencia y Tecnología (CONACYT) y la Comunidad Económica Europea (proyecto REFORLAN FP6-2004-INCODEV-3032132).

\section{LITERATURA CITADA}

Bedolla-García, B. Y., S. I. Lara-Cabrera y S. Zamudio. 2011. Dos nuevas especies de Salvia (Lamiaceae) del centro occidente de México. Acta Bot. Mex. 95: 51-63.

Bentham, G. 1832. Labiatarum genera et species. Ridgeway, Londres, UK. 783 pp.

Bentham, G. 1848. Labiatae. In: De Candolle, A. (ed.). Prodromous systematis naturalis regni vegetabilis 12. Paris, Francia. pp. 27-610.

Bentham, G. 1876. Labiatae. In: Bentham, G. y J. D. Hooker. Genera plantarum 2. Reeve and Co. Londres, UK. pp. 1160-1196.

Briquet, J. 1897. Labiatae. In: Engler, A. y K. Prantl. Die natürlichen Pflanzenfamilien nebst ihrer Gattungen und wichtigeren Arten 4. Wilhelm Engelmann. Leipzig, Alemania. pp. 183-287.

Claßen-Bockhoff, R., P. Wester y E. Tweraser. 2003. The staminal lever mechanism in Salvia L. (Lamiaceae)-a review. Pl. Biol. 5: 33-41.

Cornejo-Tenorio, G. y G. Ibarra-Manríquez. 2011. Diversidad y distribución del género Salvia (Lamiaceae) en Michoacán, México. Rev. Mex. Biodiv. 82: 1279-1296.

Domínguez-Vázquez, G., B. Berlín, A. E. Castro-Ramírez y E. I. J. Estrada-Lugo. 2002. Revisión de la diversidad y patrones de distribución de Labiatae en Chiapas. Anales Inst. Biol. Univ. Nac. Autón. México, Ser. Bot. 73: 39-80.

dos Santos, E. P. 1991. Genre Salvia L. sous-genre Calosphace (Benth.) Benth. section Nobiles (Benth.) Epl. (Labiatae). Bradea 5: 436-454.

Epling, C.1938. The California salvias. A review of Salvia, section Audibertia. Ann. Missouri Bot. Gard. 25: 95-188. 
Epling, C. 1939. A revision of Salvia subgenus Calosphace. Repert. Spec. Nov. Regni Veg. 110: 1-383.

García-Mendoza, A. J. y J. A. Meave. 2011. Diversidad florística de Oaxaca: de musgos a angiospermas (colecciones y lista de especies). Instituto de Biología, Universidad Nacional Autónoma de México. México, D.F., México. 351 pp.

Harley, R. M., S. Atkins, A. L. Budantsev, P. D. Cantino, B. J. Conn, R. Grayer, M. M. Harley, R. de Kok, T. Krestovskaja, R. Morales, A. J. Paton, O. Ryding y T. Upson. 2004. Labiatae. In: Kadereit, J. W. The families and genera of vascular plants VII. Flowering plants. Dicotyledons. Lamiales (except Acanthaceae including Avicenniaceae). Springer. Berlin, Alemania. pp. 167-275.

Jenks, A. A., J. B. Walker y S.-C. Kim. 2011. Evolution and origins of the Mazatec hallucinogenic sage, Salvia divinorum (Lamiaceae): a molecular phylogenetic approach. J. Plant Res. 124: 593-600.

Jenks, A. A., J. B. Walker y S.-C. Kim. 2013. Phylogeny of New World Salvia subgenus Calosphace (Lamiaceae) based on cpDNA (psbA-trnH) and nrDNA (ITS) sequence data. J. Plant Res. 126: 483-496.

Lindely, J. y J. Paxon. 1853. Paxton's flower garden II. Bradbury \& Evans. Londres, UK. 186 pp.

Martínez-Gordillo, M., I. Fragoso-Martínez, M. R. García-Peña y O. Montiel. 2013. Géneros de Lamiaceae de México, diversidad y endemismo. Rev. Mex. Biodiv. 84: 30-86.

Neisess, K. R. 1983. Evolution, systematics, and terpene relationships of Salvia section Audibertia. University of California. Riverside, USA. 327 pp.

Ramamoorthy, T. P. 1984. Typifications in Salvia (Lamiaceae). Taxon 33: 322-324.

Ramamoorthy, T. P. 1985. Salvia L. In: Rzedowski, J. y G. C. de Rzedowski. Flora fanerogámica del valle de México. Volumen II. Escuela Nacional de Ciencias Biológicas e Instituto de Ecología. México, D.F., México. pp. 298-310.

Ramamoorthy, T. P. 2005. Salvia L. In: Rzedowski, G. C. de y J. Rzedowski. Flora fanerogámica del Valle de México. Instituto de Ecología, A.C. y Comisión Nacional para el Conocimiento y Uso de la Biodiversidad, 2a. ed. Pátzcuaro, México. pp. 632-644.

Ramamoorthy, T. P. y M. Elliott.1998. Lamiaceae de México: diversidad, distribución, endemismo y evolución. In: Ramamoorthy, T. P., R. Bye, A. Lot y J. Fa. Diversidad biológica de México. Orígenes y distribución. Instituto de Biología. Universidad Nacional Autónoma de México. México, D.F., México. pp. 501-526.

Rodríguez-Jiménez, L. S. y J. Espinosa-Garduño. 1996. Listado florístico del estado de Michoacán, sección III (Angiospermae: Connaraceae-Myrtaceae excepto Fagaceae, Gramineae, Krameriaceae y Leguminosae). Flora del Bajío y de Regiones Adyacentes $\mathrm{X}: 1-296$.

Standley, P. C. y L. O. Williams. 1973. Labiatae. Flora of Guatemala 24(9): 237-317.

Strachan, J. L. 1982. A revision of the Salvia dorrii complex (Lamiaceae). Brittonia 34: 151-169.

Téllez V., O. 1995. Flora, vegetación y fitogeografía de Nayarit, México. Tesis de maestría. Universidad Nacional Autónoma de México. México, D.F., México. 160 pp.

Villaseñor, J. L. 2004. Los géneros de plantas vasculares de la flora de México. Bol. Soc. Bot. Méx. 75: 105-135. 
Walker, J. B. 2006. Systematics of the genus Salvia (Lamiaceae). University of WisconsinMadison. Madison, USA. 194 pp.

Walker, J. B. y W. J. Elisens. 2001. A revision of Salvia section Heterosphace (Lamiaceae) in western North America. Sida 19: 571-589.

Walker, J. B. y K. J. Sytsma. 2007. Staminal evolution in the genus Salvia (Lamiaceae): Molecular phylogenetic evidence for multiple origins of the staminal lever. Ann. Bot. 100: 375-391.

Walker, J. B., K. J. Sytsma, J. Treutlein y M. Wink. 2004. Salvia (Lamiaceae) is not monophyletic: Implications for the systematics, radiation, and ecological specializations of Salvia and tribe Mentheae. Amer. J. Bot. 91: 1115-1125.

Wester, P. y R. Claßen-Bockhoff. 2006a. Bird pollination in South African Salvia species. Flora, Morphol. Distrib. Funct. Ecol. Pl. 201: 396-406.

Wester, P. y R. Claßen-Bockhoff. 2006b. Hummingbird pollination in Salvia haenkei (Lamiaceae) lacking the typical lever mechanism. Pl. Syst. Evol. 257: 133-146.

Wester, P. y R. Claßen-Bockhoff. 2007a. Bilabiate flowers: The ultimate response to bees? Ann. Bot. 100: 361-374.

Wester, P. y R. Claßen-Bockhoff. 2007b. Floral diversity and pollen transfer mechanisms in bird-pollinated Salvia species. Ann. Bot. 100: 401-421.

Wester, P. y R. Claßen-Bockhoff. 2011. Pollination syndromes of New World Salvia species with special reference to bird pollination. Ann. Missouri Bot. Gard. 98: 101-155.

Wieczorek, J. 2001. Georeferencing guidelines. University of California. Berkeley, USA. (manisnet.org/GeorefGuide.html; acceso: 15/11/2012). 